CTP/TAMU-17/93

\title{
Four-Dimensional String/String Duality ${ }^{\dagger}$
}

\author{
M. J. Duff and Ramzi R. Khuri* \\ Center for Theoretical Physics \\ Texas A\&M University \\ College Station, TX 77843
}

\begin{abstract}
We present supersymmetric soliton solutions of the four-dimensional heterotic string corresponding to monopoles, strings and domain walls. These solutions admit the $D=10$ interpretation of a fivebrane wrapped around 5, 4 or 3 of the 6 toroidally compactified dimensions and are arguably exact to all orders in $\alpha^{\prime}$. The solitonic string solution exhibits an $S L(2, Z)$ strong/weak coupling duality which however corresponds to an $S L(2, Z)$ target space duality of the fundamental string.
\end{abstract}

$4 / 93$

$\dagger$ Work supported in part by NSF grant PHY-9106593.

* Supported by a World Laboratory Fellowship. 


\section{Introduction}

A major problem in string theory is to go beyond a weak-coupling perturbation expansion. A possible approach to this problem is provided by the string/fivebrane duality conjecture [1],2], which states that, in their critical spacetime dimension $D=10$, superstrings (extended objects with one spatial dimension) are dual to superfivebranes (extended objects with five spatial dimensions). There is now a good deal of evidence in favor of this idea, which may be divided into: Poincare duality [1], strong/weak coupling duality [28], singularity structure duality [9] and classical/quantum duality [10,11]. Most of these discussions have focused on the $D=10$ heterotic string and its dual counterpart the $D=10$ heterotic fivebrane, but in this paper we wish to examine the four-dimensional consequences.

That the field theory limit of the $D=10$ heterotic string admits as a soliton a heterotic fivebrane [1] was first pointed out by Strominger [2]. He went on to suggest a strong/weak coupling duality between the string and the fivebrane in analogy with the MontonenOlive strong/weak coupling conjecture in four-dimensional super Yang-Mills theories [12]. This strong/weak coupling was subsequently confirmed from the point of view of Poincare duality in [3]. There it was shown that just as the string loop expansion parameter is given by

$$
g(\text { string })=e^{\phi_{0}},
$$

where $\phi_{0}$ is the dilaton VEV, so the analogous fivebrane parameter is given by

$$
g(\text { fivebrane })=e^{-\phi_{0} / 3},
$$

and hence

$$
g(\text { fivebrane })=g(\text { string })^{-1 / 3} .
$$

In the same paper [2], Strominger pointed out that after toroidal compactification to four dimensions, the fivebrane would appear as either a 0-brane, a 1-brane or a 2-brane, depending in how the fivebrane wraps around the compactified directions [13 [15]. Thus it ought to be possible to find soliton solutions directly from the four-dimensional string corresponding to monopoles (0-branes), strings (1-branes) and domain walls (2-branes). Such fivebrane-inspired supersymmetric monopoles have already been found [16 18], and here we discuss the string and domain wall. (The possibility of monopole solutions in this context was anticipated in [19], where a monopole-like solution in the massless fields sector of the bosonic string was discovered.) 
To find these multi-string and multi-domain wall solutions we shall follow the procedure outlined in [16,17] for monopoles, where it was argued that exact solutions of the heterotic string could be obtained by modifying the 't Hooft ansatz for the Yang-Mills instanton. We shall present them from both the $D=10$ and $D=4$ points of view. As with the fivebrane and the monopole, there are three types of string and domain wall solutions: neutral, gauge and symmetric. Following the arguments of [5.6], all symmetric solutions correspond to $(4,4)$ supersymmetry on the worldsheet of the fundamental string and are thus presumably exact to all orders in $\alpha^{\prime}$.

Of particular interest is the solitonic string, since its couplings to the background fields of supergravity are the same as those of the fundamental string except that the dilaton/axion field $S$ is replaced by the modulus field $T$. Thus under string/fivebrane duality, the $S L(2, Z)$ strong/weak coupling duality trades places with the $S L(2, Z)$ target space duality, in accordance with recent observations of Schwarz and Sen [20] and Binétruy 21].

\section{The General Ansatz}

We first summarize the 't Hooft ansatz for the Yang-Mills instanton. Consider the four-dimensional Euclidean action

$$
S=-\frac{1}{2 g^{2}} \int d^{4} x \operatorname{Tr} F_{\mu \nu} F^{\mu \nu}, \quad \quad \mu, \nu=1,2,3,4
$$

For gauge group $S U(2)$, the fields may be written as $A_{\mu}=(g / 2 i) \sigma^{a} A_{\mu}^{a}$ and $F_{\mu \nu}=$ $(g / 2 i) \sigma^{a} F_{\mu \nu}^{a} \quad$ (where $\sigma^{a}, a=1,2,3$ are the $2 \times 2$ Pauli matrices). A self-dual solution (but not the most general one) to the equation of motion of this action is given by the 't Hooft ansatz

$$
A_{\mu}=i \bar{\Sigma}_{\mu \nu} \partial_{\nu} \ln f
$$

where $\bar{\Sigma}_{\mu \nu}=\bar{\eta}^{i \mu \nu}\left(\sigma^{i} / 2\right)$ for $i=1,2,3$, where

$$
\begin{array}{rlrl}
\bar{\eta}^{i \mu \nu}=-\bar{\eta}^{i \nu \mu} & =\epsilon^{i \mu \nu}, & \mu, \nu=1,2,3, \\
& =-\delta^{i \mu}, & \nu &
\end{array}
$$

and where $f^{-1} \square f=0$. The ansatz for the anti-self-dual solution is similar, with the $\delta$-term in (2.3) changing sign. From this ansatz, depending on how many of the four coordinates $f$ is allowed to depend and depending on whether we compactify, we shall obtain $D=10$ 
multi-fivebrane and $D=4$ multi-monopole, multi-string and multi-domain wall solutions. We will discuss these four cases in the next section. In this section, we do not specify the precise form of $f$ or the dilaton function, but show that the derivation of the solution and most of the arguments used to demonstrate the exactness of the heterotic solution are equally valid for any $f$ satisfying $f^{-1} \square f=0$.

It turns out that there is an analog to the 't Hooft ansatz for the Yang-Mills instanton in the gravitational sector of the string, namely the axionic instanton [22]. In its simplest form, this instanton appears as a solution for the massless fields of the bosonic string [19]. The identical instanton structure arises in all supersymmetric multi-fivebrane solutions [4,5], in particular in the tree-level neutral solution [4]:

$$
\begin{aligned}
g_{\mu \nu} & =e^{2 \phi} \delta_{\mu \nu} \quad \mu, \nu=1,2,3,4, \\
g_{a b} & =\eta_{a b} \quad a, b=0,5, \ldots, 9, \\
H_{\mu \nu \lambda} & = \pm 2 \epsilon_{\mu \nu \lambda \sigma} \partial^{\sigma} \phi \quad \mu, \nu, \lambda, \sigma=1,2,3,4,
\end{aligned}
$$

with $e^{-2 \phi} \square e^{2 \phi}=0$. The D'Alembertian refers to the four-dimensional subspace $\mu, \nu, \lambda, \sigma=1,2,3,4$ and $\phi$ is taken to be independent of $\left(x^{0}, x^{5}, x^{6}, x^{7}, x^{8}, x^{9}\right)$. For zero background fermionic fields the above solution breaks half the spacetime supersymmetries.

The generalized curvature of this solution was shown [19,23 to possess (anti) selfdual structure similar to that of the 't Hooft ansatz. To see this we define a generalized curvature $\hat{R}_{\nu \rho \sigma}^{\mu}$ in terms of the standard curvature $R^{\mu}{ }_{\nu \rho \sigma}$ and $H_{\mu \alpha \beta}$ :

$$
\hat{R}_{\nu \rho \sigma}^{\mu}=R_{\nu \rho \sigma}^{\mu}+\frac{1}{2}\left(\nabla_{\sigma} H_{\nu \rho}^{\mu}-\nabla_{\rho} H_{\nu \sigma}^{\mu}\right)+\frac{1}{4}\left(H_{\nu \rho}^{\lambda} H_{\sigma \lambda}^{\mu}-H_{\nu \sigma}^{\lambda} H_{\rho \lambda}^{\mu}\right) .
$$

One can also define $\hat{R}_{\nu \rho \sigma}^{\mu}$ as the Riemann tensor generated by the generalized Christoffel symbols $\hat{\Gamma}_{\alpha \beta}^{\mu}$, where $\hat{\Gamma}_{\alpha \beta}^{\mu}=\Gamma_{\alpha \beta}^{\mu}-(1 / 2) H^{\mu}{ }_{\alpha \beta}$. The crucial observation for obtaining higher-loop and even exact solutions is the following. For any solution given by (2.4), we can express the generalized curvature in terms of the dilaton field as [19]

$$
\begin{aligned}
\hat{R}_{\nu \rho \sigma}^{\mu} & =\delta_{\mu \sigma} \nabla_{\rho} \nabla_{\nu} \phi-\delta_{\mu \rho} \nabla_{\sigma} \nabla_{\nu} \phi+\delta_{\nu \rho} \nabla_{\sigma} \nabla_{\mu} \phi-\delta_{\nu \sigma} \nabla_{\rho} \nabla_{\mu} \phi \\
& \pm \epsilon_{\mu \nu \rho \lambda} \nabla_{\sigma} \nabla_{\lambda} \phi \mp \epsilon_{\mu \nu \sigma \lambda} \nabla_{\rho} \nabla_{\lambda} \phi
\end{aligned}
$$

It easily follows that

$$
\hat{R}_{\nu \rho \sigma}^{\mu}=\mp \frac{1}{2} \epsilon_{\rho \sigma}{ }^{\lambda \gamma} \hat{R}_{\nu \lambda \gamma}^{\mu}
$$

So the (anti) self-duality appears in the gravitational sector of the string in terms of its generalized curvature. 
We now turn to the exact heterotic solution. The tree-level supersymmetric vacuum equations for the heterotic string are given by

$$
\begin{aligned}
\delta \psi_{M} & =\left(\nabla_{M}-\frac{1}{4} H_{M A B} \Gamma^{A B}\right) \epsilon=0, \\
\delta \lambda & =\left(\Gamma^{A} \partial_{A} \phi-\frac{1}{6} H_{A B C} \Gamma^{A B C}\right) \epsilon=0, \\
\delta \chi & =F_{A B} \Gamma^{A B} \epsilon=0
\end{aligned}
$$

where $A, B, C, M=0,1,2, \ldots, 9$ and where $\psi_{M}, \lambda$ and $\chi$ are the gravitino, dilatino and gaugino fields. The Bianchi identity is given by

$$
d H=\frac{\alpha^{\prime}}{4}\left(\operatorname{tr} R \wedge R-\frac{1}{30} \operatorname{Tr} F \wedge F\right) .
$$

The $(9+1)$-dimensional Majorana-Weyl fermions decompose into chiral spinors according to $S O(9,1) \supset S O(5,1) \otimes S O(4)$ for the $M^{9,1} \rightarrow M^{5,1} \times M^{4}$ decomposition. Then (2.4) with arbitrary dilaton and with constant chiral spinors $\epsilon_{ \pm}$solves the supersymmetry equations with zero background fermi fields provided the YM gauge field satisfies the instanton (anti) self-duality condition [2]

$$
F_{\mu \nu}= \pm \frac{1}{2} \epsilon_{\mu \nu}^{\lambda \sigma} F_{\lambda \sigma}
$$

In the absence of a gauge sector, the multi-fivebrane solution is identical to the "neutral" tree-level solution shown in (2.4). A perturbative "gauge" fivebrane solution was found in [2]. An exact solution is obtained as follows. Define a generalized connection by

$$
\Omega_{ \pm M}^{A B}=\omega_{M}^{A B} \pm H_{M}^{A B}
$$

in an $\mathrm{SU}(2)$ subgroup of the gauge group, and equate it to the gauge connection $A_{\mu}$ [24] so that the corresponding curvature $R\left(\Omega_{ \pm}\right)$cancels against the Yang-Mills field strength $F$ and $d H=0$. For $e^{-2 \phi} \square e^{2 \phi}=0$ (or $e^{2 \phi}=e^{2 \phi_{0}} f$ ) the curvature of the generalized connection can be written in terms of the dilaton as in (2.6) from which it follows that both $F$ and $R$ are (anti) self-dual. This solution becomes exact since $A_{\mu}=\Omega_{ \pm \mu}$ implies that all the higher order corrections vanish [5]. The self-dual solution for the gauge connection is then given by the 't Hooft ansatz. So the heterotic solution combines a YM instanton in the gauge sector with an axionic instanton in the gravity sector. In addition, the heterotic solution has finite action. Further arguments supporting the exactness of this solution based on $(4,4)$ worldsheet supersymmetry are shown in [5]. Note that at no point in this discussion do we refer to the specific form of $f$, so that all of the above arguments apply for an arbitrary solution of $f^{-1} \square f=0$. 


\section{Monopoles, Strings and Domain Walls}

We now go back to the 't Hooft ansatz (2.1)-(2.3) and solve the equation $f^{-1} \square f=0$. If we take $f$ to depend on all four coordinates we obtain a multi-instanton solution

$$
f_{I}=1+\sum_{i=1}^{N} \frac{\rho_{i}^{2}}{\left|\vec{x}-\vec{a}_{i}\right|^{2}}
$$

where $\rho_{i}^{2}$ is the instanton scale size and $\vec{a}_{i}$ the location in four-space of the $i$ th instanton. For $e^{2 \phi}=e^{2 \phi_{0}} f_{I}$, and assuming no dimensions are compactified, we obtain from (2.4) the neutral fivebrane of [4] and the exact heterotic fivebrane of [5,6] in $D=10$. The solitonic fivebrane tension $\widetilde{T_{6}}$ is related to the fundamental string tension $T_{2}\left(=1 / 2 \pi \alpha^{\prime}\right)$ by the Dirac quantization condition [3]

$$
\kappa_{10}^{2} \widetilde{T_{6}} T_{2}=n \pi
$$

where $n$ is an integer and where $\kappa_{10}^{2}$ is the $D=10$ gravitational constant. This implies $\rho_{i}^{2}=e^{-2 \phi_{0}} n_{i} \alpha^{\prime}$, where $n_{i}$ are integers. Near each source the solution is described by an exact conformal field theory [22, 19,5].

Instead, let us single out a direction in the transverse four-space (say $x^{4}$ ) and assume all fields are independent of this coordinate. Since all fields are already independent of $x^{5}, x^{6}, x^{7}, x^{8}, x^{9}$, we may consistently assume the $x^{4}, x^{5}, x^{6}, x^{7}, x^{8}, x^{9}$ are compactified on a six-dimensional torus, where we shall take the $x^{4}$ circle to have circumference $L e^{-\phi_{0}}$ and the rest to have circumference $L$, so that $\kappa_{4}^{2}=\kappa_{10}^{2} e^{\phi_{0}} / L^{6}$. Then the solution for $f$ satisfying $f^{-1} \square f=0$ has multi-monopole structure

$$
f_{M}=1+\sum_{i=1}^{N} \frac{m_{i}}{\left|\vec{x}-\vec{a}_{i}\right|}
$$

where $m_{i}$ is proportional to the charge and $\vec{a}_{i}$ the location in the three-space (123) of the $i$ th monopole. If we make the identification $\Phi \equiv A_{4}$ then the lagrangian density may be rewritten as

$$
F_{\mu \nu}^{a} F_{\mu \nu}^{a}=F_{j k}^{a} F_{j k}^{a}+2 F_{k 4}^{a} F_{k 4}^{a}=F_{j k}^{a} F_{j k}^{a}+2 D_{k} \Phi^{a} D_{k} \Phi^{a}
$$

where $j, k=1,2,3$. We now go to $3+1$ space (0123) with the Lagrangian density

$$
\mathcal{L}=-\frac{1}{4} G_{\alpha \beta}^{a} G^{\alpha \beta a}-\frac{1}{2} D_{\alpha} \Phi^{a} D^{\alpha} \Phi^{a}
$$


where $\alpha, \beta=0,1,2,3$. It follows that the above multi-monopole ansatz is a static solution with $A_{0}^{a}=0$ and all time derivatives vanish. The solution in $3+1$ dimensions has the form

$$
\begin{aligned}
\Phi^{a} & =\mp \frac{1}{g} \delta^{a j} \partial_{j} \omega, \\
A_{k}^{a} & =\frac{1}{g} \epsilon^{a k j} \partial_{j} \omega,
\end{aligned}
$$

where $j, k=1,2,3$ and where $\omega \equiv \ln f$ and $g$ is the YM coupling constant. This solution represents a multi-monopole configuration with sources at $\vec{a}_{i}, i=1,2 \ldots N$ [16, 17]. For $e^{2 \phi}=e^{2 \phi_{0}} f_{M}$, we obtain from (2.4) a neutral monopole solution and the exact heterotic monopole solution of [16,17]. The monopole strength is given by $\tilde{g}=\sqrt{2} \kappa_{4} \widetilde{T_{1}}$, where $\widetilde{T_{1}}=\widetilde{T_{6}} L^{5}$ obeys, from $(\widetilde{3.2})$, the quantization condition

$$
e^{-\phi_{0}} \kappa_{4}^{2} T_{2} \widetilde{T_{1}}=\frac{n \pi}{L}
$$

This implies $m_{i}=e^{-\phi_{0}} n_{i} \pi \alpha^{\prime} / L$. Similarly the "electric" charge of the fundamental string is $e=\sqrt{2} \kappa_{4} T_{1}$, where $T_{1}=T_{2} L e^{-\phi_{0}}$, and hence

$$
e \tilde{g}=2 \pi n
$$

as expected. Unlike for the instanton, in the monopole case we cannot identify the explicit coset conformal field theory near each source. A noteworthy feature of this solution is that the divergences from both gauge and gravitational sectors cancel to yield a finite lagrangian, and finite soliton mass.

It is straightforward to reduce the multi-monopole solution to an explicit solution in the four-dimensional space (0123). The gauge field reduction is exactly as above, i.e. we replace $A_{4}$ with the scalar field $\Phi$. In the gravitational sector, the reduction from ten to five dimensions is trivial, as the metric is flat in the subspace (56789). In going from five to four dimensions, one follows the usual Kaluza-Klein procedure of replacing $g_{44}$ with a scalar field $e^{-2 \sigma}$. The tree-level effective action reduces in four dimensions to

$$
S_{4}=\frac{1}{2 \kappa_{4}^{2}} \int d^{4} x \sqrt{-g} e^{-2 \phi-\sigma}\left(R+4(\partial \phi)^{2}+4 \partial \sigma \cdot \partial \phi-e^{2 \sigma} \frac{M_{\alpha \beta} M^{\alpha \beta}}{4}\right),
$$

where $\alpha, \beta=0,1,2,3$, where $M_{\alpha \beta}=H_{\alpha \beta}=\partial_{\alpha} B_{\beta}-\partial_{\beta} B_{\alpha}$, and where $B_{\alpha}=B_{\alpha 4}$. The four-dimensional monopole solution for this reduced action is then given by

$$
\begin{aligned}
e^{2 \phi}=e^{-2 \sigma} & =e^{2 \phi_{0}}\left(1+\sum_{i=1}^{N} \frac{m_{i}}{\left|\vec{x}-\vec{a}_{i}\right|}\right) \\
d s^{2} & =-d t^{2}+e^{2 \phi}\left(d x_{1}^{2}+d x_{2}^{2}+d x_{3}^{2}\right) \\
M_{i j} & = \pm \epsilon_{i j k} \partial_{k} e^{2 \phi}, \quad i, j, k=1,2,3 .
\end{aligned}
$$


Since the tree-level solution is exact, we need not reduce the higher order corrections to the action.

We now modify the solution of the 't Hooft ansatz even further and choose two directions in the four-space (1234) (say $x^{3}$ and $x^{4}$ ) and assume all fields are independent of both of these coordinates. We may now consistently assume that $x^{3}, x^{4}, x^{6}, x^{7}, x^{8}, x^{9}$ are compactified on a six-dimensional torus, where we shall take the $x^{3}$ and $x^{4}$ circles to have circumference $L e^{-\phi_{0}}$ and the remainder to have circumference $L$, so that $\kappa_{4}^{2}=\kappa_{10}^{2} e^{2 \phi_{0}} / L^{6}$. Then the solution for $f$ satisfying $f^{-1} \square f=0$ has multi-string structure

$$
f_{S}=1-\sum_{i=1}^{N} \lambda_{i} \ln \left|\vec{x}-\vec{a}_{i}\right|
$$

where $\lambda_{i}$ is the charge per unit length and $\vec{a}_{i}$ the location in the two-space (12) of the $i$ th string. If we make the identification $\Phi \equiv A_{4}$ and $\Psi \equiv A_{3}$ then the lagrangian density for the above ansatz can be rewritten as

$$
F_{\mu \nu}^{a} F_{\mu \nu}^{a}=F_{j k}^{a} F_{j k}^{a}+2 D_{k} \Phi^{a} D_{k} \Phi^{a}+2 D_{k} \Psi^{a} D_{k} \Psi^{a}
$$

where $j, k=1,2$. We now go to the $3+1$ space (0125) with the lagrangian density

$$
\mathcal{L}=-\frac{1}{4} G_{\rho \sigma}^{a} G^{\rho \sigma a}-\frac{1}{2} D_{\rho} \Phi^{a} D^{\rho} \Phi^{a}-\frac{1}{2} D_{\rho} \Psi^{a} D^{\rho} \Psi^{a},
$$

where $\rho, \sigma=0,1,2,5$. It follows that the multi-string ansatz is a static solution with $A_{0}^{a}=0$ and all time derivatives vanish. The solution in $3+1$ dimensions has the form

$$
\begin{aligned}
\Phi^{a} & =\mp \frac{1}{g} \delta^{a j} \partial_{j} \omega, \\
\Psi^{k} & =\frac{1}{g} \epsilon^{k j} \partial_{j} \omega, \\
A_{k}^{a} & =-\delta^{a 3} \frac{1}{g} \epsilon^{k j} \partial_{j} \omega,
\end{aligned}
$$

where $j, k=1,2$ and where $\omega \equiv \ln f$. This solution represents a multi-string configuration with sources at $\vec{a}_{i}, i=1,2 \ldots N$. By setting $e^{2 \phi}=e^{2 \phi_{0}} f_{S}$, we obtain from (2.4) a neutral multi-string solution and an exact heterotic multi-string solution. The solitonic string tension $\widetilde{T_{2}}$ is given by $\widetilde{T_{6}} L^{4}$ and from (3.2) is related to the fundamental string tension $T_{2}$ by

$$
e^{-2 \phi_{0}} \kappa_{4}^{2} T_{2} \widetilde{T_{2}}=\frac{n \pi}{L^{2}}
$$


This implies $\lambda_{i}=n_{i} 2 \pi \alpha^{\prime} / L^{2}$. Like the monopole and unlike the instanton, we cannot identify an explicit coset conformal field theory near each source. Also like the monopole, the lagrangian per unit length for the string solution is finite as a result of the cancellation of divergences between the gauge and gravitational sectors.

As in the multi-monopole case, it is straightforward to reduce the multi-string solution to a solution in the four-dimensional space (0125). The gauge field reduction is done in (3.14). In the gravitational sector, the reduction from ten to six dimensions is trivial, as the metric is flat in the subspace (6789). In going from six to four dimensions, we compactify the $x_{3}$ and $x_{4}$ directions and again follow the Kaluza-Klein procedure by replacing $g_{33}$ and $g_{44}$ with a scalar field $e^{-2 \sigma}$. The tree-level effective action reduces in four dimensions to

$$
S_{4}=\frac{1}{2 \kappa_{4}^{2}} \int d^{4} x \sqrt{-g} e^{-2 \phi-2 \sigma}\left(R+4(\partial \phi)^{2}+8 \partial \sigma \cdot \partial \phi+2(\partial \sigma)^{2}-e^{4 \sigma} \frac{N_{\rho} N^{\rho}}{2}\right),
$$

where $\rho=0,1,2,5$, where $N_{\rho}=H_{\rho 34}=\partial_{\rho} B$, and where $B=B_{34}$. The four-dimensional string soliton solution for this reduced action is then given by

$$
\begin{aligned}
e^{2 \phi}=e^{-2 \sigma} & =e^{2 \phi_{0}}\left(1-\sum_{i=1}^{N} \lambda_{i} \ln \left|\vec{x}-\vec{a}_{i}\right|\right) \\
d s^{2} & =-d t^{2}+d x_{5}^{2}+e^{2 \phi}\left(d x_{1}^{2}+d x_{2}^{2}\right) \\
N_{i} & = \pm \epsilon_{i j} \partial_{j} e^{2 \phi}
\end{aligned}
$$

Again since the tree-level solution is exact, we do not bother to reduce the higher order corrections to the action.

We complete the family of solitons that can be obtained from the solutions of the 't Hooft ansatz by demanding that $f$ depend on only one coordinate, say $x^{1}$. We may now consistently assume that $x^{2}, x^{3}, x^{4}, x^{7}, x^{8}, x^{9}$ are compactified on a six-dimensional torus, where we shall take the $x^{2}, x^{3}$ and $x^{4}$ circles to have circumference $L e^{-\phi_{0}}$ and the rest to have circumference $\kappa_{4}^{2}=\kappa_{10}^{2} e^{3 \phi_{0}} / L^{6}$. Then the solution of $f^{-1} \square f=0$ has domain wall structure with the "confining potential"

$$
f_{D}=1+\sum_{i=1}^{N} \Lambda_{i}\left|x_{1}-a_{i}\right|
$$

where $\Lambda_{i}$ are constants. By setting $e^{2 \phi}=e^{2 \phi_{0}} f_{D}$, we obtain from (2.4) a neutral domain wall solution and an exact heterotic domain wall solution. The solitonic domain wall 
tension $\widetilde{T_{3}}$ is given by $\widetilde{T_{6}} L^{3}$ and from $(3.2)$ is related to the fundamental string tension $T_{2}$ by

$$
e^{-3 \phi_{0}} \kappa_{4}^{2} T_{2} \widetilde{T_{3}}=\frac{n \pi}{L^{3}}
$$

This implies $\Lambda_{i}=e^{\phi_{0}} n_{i}(2 \pi)^{2} \alpha^{\prime} / L^{3}$. Like the monopole and string we cannot identify an explicit coset conformal field theory near each source. Again the reduction to $D=4$ is straightforward. In the gauge sector, the action reduces to YM + three scalar fields $\Phi, \Psi$ and $\Pi$. For the spacetime (0156) the solution for the fields is given by

$$
\begin{aligned}
& \Phi^{1}=\mp \frac{\Lambda}{g\left(1+\Lambda\left|x_{1}\right|\right)}, \\
& \Psi^{3}=\frac{\Lambda}{g\left(1+\Lambda\left|x_{1}\right|\right)}, \\
& \Pi^{2}=-\frac{\Lambda}{g\left(1+\Lambda\left|x_{1}\right|\right)}, \\
& A_{\mu}=0
\end{aligned}
$$

where $\mu=0,1,5,6$. In the gravitational sector the tree-level effective action in $D=4$ has the form

$$
S_{4}=\frac{1}{2 \kappa^{2}} \int d^{4} x \sqrt{-g} e^{-2 \phi-3 \sigma}\left(R+4(\partial \phi)^{2}+12 \partial \sigma \cdot \partial \phi+6(\partial \sigma)^{2}-e^{6 \sigma} \frac{P^{2}}{2}\right)
$$

where $P=H_{234}$. The four-dimensional domain wall solution for this reduced action is then given by

$$
\begin{aligned}
e^{2 \phi}=e^{-2 \sigma} & =e^{2 \phi_{0}}\left(1+\Lambda\left|x_{1}\right|\right), \\
d s^{2} & =-d t^{2}+d x_{5}^{2}+d x_{6}^{2}+e^{2 \phi} d x_{1}^{2}, \\
P & =\Lambda\left(\Theta\left(x_{1}\right)-\Theta\left(-x_{1}\right)\right) .
\end{aligned}
$$

Again since the tree-level solution is exact, we do not bother to reduce the higher order corrections to the action. A trivial change of coordinates reveals that the spacetime is, in fact, flat. Dilaton domain walls with a flat spacetime have recently been discussed in a somewhat different context in [25,26].

As for the fivebrane in $D=10$, the mass of the monopole, the mass per unit length of the string and the mass per unit area of the domain wall saturate a Bogomol'nyi bound with the topological charge. (In the case of the string and domain, wall, however, we must follow [27] and extrapolate the meaning of the ADM mass to non-asymptotically flat spacetimes.) 


\section{String/String Duality}

Let us focus on the solitonic string configuration (3.17) in the case of a single source. In terms of the complex field

$$
\begin{aligned}
T & =T_{1}+i T_{2} \\
& =B_{34}+i e^{-2 \sigma} \\
& =B_{34}+i \sqrt{\operatorname{det} g_{m n}^{S}} \quad m, n=3,4,6,7,8,9
\end{aligned}
$$

where $g_{M N}^{S}$ is the string $\sigma$-model metric, the solution takes the form (with $z=x_{1}+x_{2}$ )

$$
\begin{aligned}
T & =\frac{1}{2 \pi i} \ln \frac{z}{r_{0}} \\
d s^{2} & =-d t^{2}+d x_{5}^{2}-\frac{1}{2 \pi} \ln \frac{r}{r_{0}} d z d \bar{z}
\end{aligned}
$$

whereas both the four-dimensional (shifted) dilaton $\eta=\phi+\sigma$ and the four-dimensional two-form $B_{\mu \nu}$ are zero. In terms of the canonical metric $g_{\mu \nu}, T_{1}$ and $T_{2}$, the relevant part of the action takes the form

$$
S_{4}=\frac{1}{2 \kappa_{4}^{2}} \int d^{4} x \sqrt{-g}\left(R-\frac{1}{2 T_{2}^{2}} g^{\mu \nu} \partial_{\mu} T \partial_{\nu} \bar{T}\right)
$$

and is invariant under the $S L(2, R)$ transformation

$$
T \rightarrow \frac{a T+b}{c T+d}, \quad a d-b c=1
$$

The discrete subgroup $S L(2, Z)$, for which $a, b, c$ and $d$ are integers, is just a subgroup of the $O(6,22 ; Z)$ target space duality, which can be shown to be an exact symmetry of the compactified string theory at each order of the string loop perturbation expansion.

This $S L(2, Z)$ is to be contrasted with the $S L(2, Z)$ symmetry of the elmentary fourdimensional solution of Dabholkar et al. [27]. In their solution $T_{1}$ and $T_{2}$ are zero, but $\eta$ and $B_{\mu \nu}$ are non-zero. The relevant part of the action is

$$
S_{4}=\frac{1}{2 \kappa_{4}^{2}} \int d^{4} x \sqrt{-g}\left(R-2 g^{\mu \nu} \partial_{\mu} \eta \partial_{\nu} \eta-\frac{1}{12} e^{-4 \eta} H_{\mu \nu \rho} H^{\mu \nu \rho}\right) .
$$

The equations of motion of this theory also display an $S L(2, R)$ symmetry, but this becomes manifest only after dualizing and introducing the axion field $a$ via

$$
\sqrt{-g} g^{\mu \nu} \partial_{\nu} a=\frac{1}{3 !} \epsilon^{\mu \nu \rho \sigma} H_{\nu \rho \sigma} e^{-4 \eta}
$$


Then in terms of the complex field

$$
\begin{aligned}
S & =S_{1}+i S_{2} \\
& =a+i e^{-2 \eta}
\end{aligned}
$$

the Dabholkar et al. fundamental string solution may be written

$$
\begin{aligned}
S & =\frac{1}{2 \pi i} \ln \frac{z}{r_{0}}, \\
d s^{2} & =-d t^{2}+d x_{5}^{2}-\frac{1}{2 \pi} \ln \frac{r}{r_{0}} d z d \bar{z} .
\end{aligned}
$$

Thus (4.2) and (4.8) are the same with the replacement $T \leftrightarrow S$. It has been conjectured that this second $S L(2, Z)$ symmetry may also be a symmetry of string theory [28,29, 30, but this is far from obvious order by order in the string loop expansion since it involves a strong/weak coupling duality $\eta \rightarrow-\eta$. What interpretation are we to give to these two $S L(2, Z)$ symmetries: one an obvious symmetry of the fundamental string and the other an obscure symmetry of the fundamental string?

While the present work was in progress, we became aware of recent interesting papers by Sen [31], Schwarz and Sen [20] and Binétruy [21]. In particular, Sen draws attention to the Dabholkar et al. string solution (4.8) and its associated $S L(2, Z)$ symmetry as supporting evidence in favor of the conjecture that $S L(2, Z)$ invariance may indeed be an exact symmetry of string theory. He also notes that the spectrum of electric and magnetic charges is consistent with the proposed $S L(2, Z)$ symmetry [31. ${ }^{\dagger}$

All of these observations fall into place if one accepts the proposal of Schwarz and Sen [20]: under string/fivebrane duality the roles of the target-space duality and the strong/weak coupling duality are interchanged! This proposal is entirely consistent with an earlier one that under string/fivebrane duality the roles of the $\sigma$-model loop expansion and the string loop expansion are interchanged [10]. In this light, the two $S L(2, Z)$ symmetries discussed above are just what one expects. From the string point of view, the $T$-field $S L(2, Z)$ is an obvious target space symmetry, manifest order by order in string loops whereas the $S$-field $S L(2, Z)$ is an obscure strong/weak coupling symmetry. From the fivebrane point of view, it is the $T$-field $S L(2, Z)$ which is obscure while the $S$-field $S L(2, Z)$ is an "obvious" target

$\dagger$ Sen also discusses the concept of a "dual string", but for him this is obtained from the fundamental string by an $S L(2, Z)$ transform. For us, a dual string is obtained by the replacement $S \leftrightarrow T$. 
space symmetry. (This has not yet been proved except at the level of the low-energy field theory, however. It would be interesting to have a proof starting from the worldvolume of the fivebrane.) This interchange in the roles of the $S$ and $T$ field in going from the string to the fivebrane has also been noted by Binétruy [21]. It is made more explicit when $S$ is expressed in terms of the variables appearing naturally in the fivebrane version

$$
\begin{aligned}
S & =S_{1}+i S_{2} \\
& =a_{056789}+i e^{-2 \eta}, \\
& =a_{056789}+i \sqrt{\operatorname{det} g_{m n}^{F}}, \quad m, n=3,4,6,7,8,9,
\end{aligned}
$$

where $g_{M N}^{F}=e^{-\phi / 3} g_{M N}^{S}$ is the fivebrane $\sigma$-model metric [3] and $a_{M N P Q R S}$ is the 6-form which couples to the 6-dimensional worldvolume of the fivebrane, in complete analogy with (4.1).

Note, however, that unlike the Dabholkar et al. solution, our symmetric solution (3.14) also involves the non-abelian gauge fields $A_{\rho}, \Phi, \Psi$ whose interactions appear to destroy the $S L(2, Z)$. This remains a puzzle. (A generalization of the $D=4$ Dabholkar et al. solution involving gauge fields may also be possible by obtaining it as a soliton of the fivebrane theory. This would involve a $D=4$ analogue of the $D=10$ solution discussed in $\| 7$.

\section{Discussion}

It may at first sight seem strange that a string can be dual to another string in $D=4$. After all, the usual formula relating the dimension of an extended object, $d$, to that of the dual object, $\tilde{d}$, is $\tilde{d}=D-d-2$. So one might expect string/string duality only in $D=6$ [10]. However, when we compactify $n$ dimensions and allow the dual object to wrap around

$m \leq d-1$ of the compactified directions we find $\tilde{d}_{\text {effective }}=\tilde{d}-m=D_{\text {effective }}-d-2+(n-m)$, where $D_{\text {effective }}=D-n$. In particular for $D_{\text {effective }}=4, d=2, n=6$ and $m=4$, we find $\tilde{d}_{\text {effective }}=2$.

Thus the whole string/fivebrane duality conjecture is put in a different light when viewed from four dimensions. After all, our understanding of the quantum theory of fivebranes in $D=10$ is rather poor, whereas the quantum theory of strings in $D=4$ is comparatively well-understood (although we still have to worry about the monopoles and domain walls). In particular, the dual string will presumably exhibit the normal kind of mass 
spectrum with linearly rising Regge trajectories, since the classical ( $\hbar$-independent) string expression $\widetilde{T}_{6} L^{4} \times(\text { angular momentum) has dimensions of (mass })^{2}$, whereas the analogous classical expression for an uncompactified fivebrane is $\left(\widetilde{T}_{6}\right)^{1 / 5} \times($ angular momentum) which has dimensions $(\text { mass })^{6 / 5}[\mathbb{[}]$. Indeed, together with the observation that the $S L(2, Z)$ strong/weak coupling duality appears only after compactifying at least 6 dimensions, it is tempting to revive the earlier conjecture [1, 15] that the internal consistency of the fivebrane may actually require compactification.

\section{Acknowledgements}

We would like to thank Pierre Binétruy, Ruben Minasian, Joachim Rahmfeld, John Schwarz and Ashoke Sen for helpful discussions. 


\section{References}

[1] M. J. Duff, Class. Quant. Grav. 5 (1988).

[2] A. Strominger, Nucl. Phys. B343 (1990) 167.

[3] M. J. Duff and J. X. Lu, Nucl. Phys. B354 (1991) 129.

[4] M. J. Duff and J. X. Lu, Nucl. Phys. B354 (1991) 141.

[5] C. G. Callan, J. A. Harvey and A. Strominger, Nucl. Phys. B359 (1991) 611.

[6] C. G. Callan, J. A. Harvey and A. Strominger, Nucl. Phys. B367 (1991) 60.

[7] M. J. Duff and J. X. Lu, Phys. Rev. Lett. 66 (1991) 1402.

[8] M. J. Duff and J. X. Lu, Class. Quant. Gravity 9 (1992) 1.

[9] M. J. Duff, R. R. Khuri and J. X. Lu, Nucl. Phys. B377 (1992) 281.

[10] M. J. Duff and J. X. Lu, Nucl. Phys. B357 (1991) 534.

[11] J. A. Dixon, M. J. Duff and J. C. Plefka, Phys. Rev. Lett. 69 (1992) 3009.

[12] C. Montonen and D. Olive, Phys. Lett. B72 (1977) 117.

[13] M. J. Duff, P. Howe, T. Inami and K. S. Stelle, Phys. Lett. B191 (1987) 70.

[14] M. J. Duff, T. Inami, C. N. Pope, E. Sezgin and K. S. Stelle, Nucl. Phys. B297 (1988) 515.

[15] K. Fujikawa and J. Kubo, Nucl. Phys. B356 (1991) 208.

[16] R. R. Khuri, Phys. Lett. B294 (1992) 325.

[17] R. R. Khuri, Nucl. Phys. B387 (1992) 315.

[18] J. P. Gauntlett, J. A. Harvey and J. T. Liu, EFI-92-67, IFP-434-UNC.

[19] R. R. Khuri, Phys. Lett. B259 (1991) 261.

[20] J. H. Schwarz and A. Sen, NSF-ITP-93-46, CALT-68-1863, TIFR-TH-93-19.

[21] P. Binétruy, NSF-ITP-93-60.

[22] S. J. Rey, Phys. Rev. D43 (1991) 526.

[23] R. R. Khuri, Phys. Rev. D46 (1992) 4526.

[24] J. M. Charap and M. J. Duff, Phys. Lett. B69 (1977) 445.

[25] H. La, CTP-TAMU-52/92.

[26] M. Cvetič, UPR-560-T.

[27] A. Dabholkar, G. Gibbons, J. A. Harvey and F. Ruiz Ruiz, Nucl. Phys. B340 (1990) 33.

[28] A. Font, L. Ibáñez, D. Lust and F. Quevedo, Phys. Lett. B249 (1990) 35.

[29] A. Sen, TIFR-TH-92-41.

[30] J. Schwarz, CALT-68-1815.

[31] A. Sen, TIFR-TH-93-03. 